\title{
Quantitative Determination of Chemical Composition of Multinary III/V Semiconductors With Sublattice Resolution Using Aberration Corrected HAADF-STEM
}

\author{
Andreas Beyer ${ }^{1}$, Nikolai Knaub ${ }^{1}$ and Kerstin Volz ${ }^{1}$ \\ 1. Philipps-Universität Marburg, Faculty of Physics and Materials Science Center, 35032 Marburg, \\ Germany
}

Multinary III/V materials such as Ga(NAsP) or (GaIn)(NAs) have a great potential for applications like lasers or solar cells [1]. This is mainly due to the independent tunability of the bandgap and the lattice constant by intentionally changing the composition of the group III and the group V sublattice, respectively.

Methods to determine the actual chemical composition of a multinary material often require rather crude assumptions or the combination of several techniques e.g. high angle annular dark field (HAADF) imaging and strain state analysis in scanning transmission electron microscopy (STEM) [2]. Aberration corrected STEM under HAADF conditions facilitates atomic resolution imaging and therefore allows to visualize the individual sublattices. Here we choose GaP, GaAs and their ternary alloy $\mathrm{Ga}(\mathrm{PAs})$ as a model system to investigate the influence of the chemical composition on the HAADF intensity for each sublattice independently.

Epitaxial $\mathrm{GaP}, \mathrm{Ga}(\mathrm{PAs})$ and $\mathrm{GaAs}$ layers were grown via metal organic vapor phase epitaxy on $\mathrm{GaP}$ and GaAs substrates, respectively. Electron transparent samples were prepared by conventional mechanical grinding followed by argon ion milling in a Gatan PIPS. For the TEM investigation, a $<010>$ zone axis was chosen as it exhibits the maximum spatial separation of the individual sublattices. The characterization was performed using an aberration corrected JEOL JEM 2200 FS operating at $200 \mathrm{kV}$. Complementary frozen phonon simulations were carried out using the STEMSIM code [3].

Figure 1 shows HAADF measurements of $\mathrm{GaP}(\mathrm{a}), \mathrm{Ga}\left(\mathrm{P}_{0.95} \mathrm{As}_{0.05}\right)$ (b) and $\mathrm{GaAs}(\mathrm{c})$ samples with comparable thicknesses. A thickness in the range of $30 \mathrm{~nm}$ was determined by comparison of the measured intensity to simulated one. Figure 2 depicts the statistical evaluation of these samples, it was derived by procedure described in the following. In each image the individual column positions were determined with subpixel accuracy using the peak pairs code [4]. Utilizing an in house written Matlab code, the two sublattices were separated from each other and the intensity around a peak position was integrated. An integration radius $1 / 3$ of the average next neighbor distance was chosen as this value shows the best tradeoff between a reduction of experimental noise and a loose of spatial information. Finally the measured intensities of the columns are drawn versus their relative frequency for the group V (Fig. 2 (a)) and the group III sublattice (Fig. 2 (b)), respectively (GaP in red, $\mathrm{Ga}$ (PAs) in green and GaAs in blue). As expected from the Z-dependence of the HAADF imaging, a shift of the group $\mathrm{V}$ values towards higher intensities is visible with increasing fraction of As. Moreover, the group III columns show a dependence on the As content as well, although they are occupied with Ga only. We attribute this behavior to a "crosstalk" of the individual sublattices caused by the finite size of the electron probe. Nevertheless, the dependence of the intensity on the composition is much more prominent on the partially occupied group V lattice. The behavior on both lattices is in agreement with simulations, if the microscope parameters, especially the actual probe size, are taken into account. Utilizing this statistical approach the influence of chemical composition 
and the crosstalk of one column to another can be quantified separately. This facilitates the quantitative evaluation of quarternary systems with atomic resolution as well.

This contribution will show a method how aberration corrected STEM can be used to determine the composition of the sublattices of multinary III/V systems [5].

\section{References:}

[1] B. Kunert, K. Volz, J. Koch, and W. Stolz, Appl. Phys. Lett., 88 (2006), , p. 182108.

[2] T. Grieb et al., Microsc. Microanal, 20, (2014), pp. 1740-52.

[3] A. Rosenauer and M. Schowalter, Microsc. Semicond. Mater. 2007, 120, (2008), pp. 170-172.

[4] P. L. Galindo et al., Ultramicroscopy, 107, (2007), pp. 1186-93.

[5] The authors acknowledge funding from the DFG in the framework of GRK 1782 (functionalisation of semiconductors).
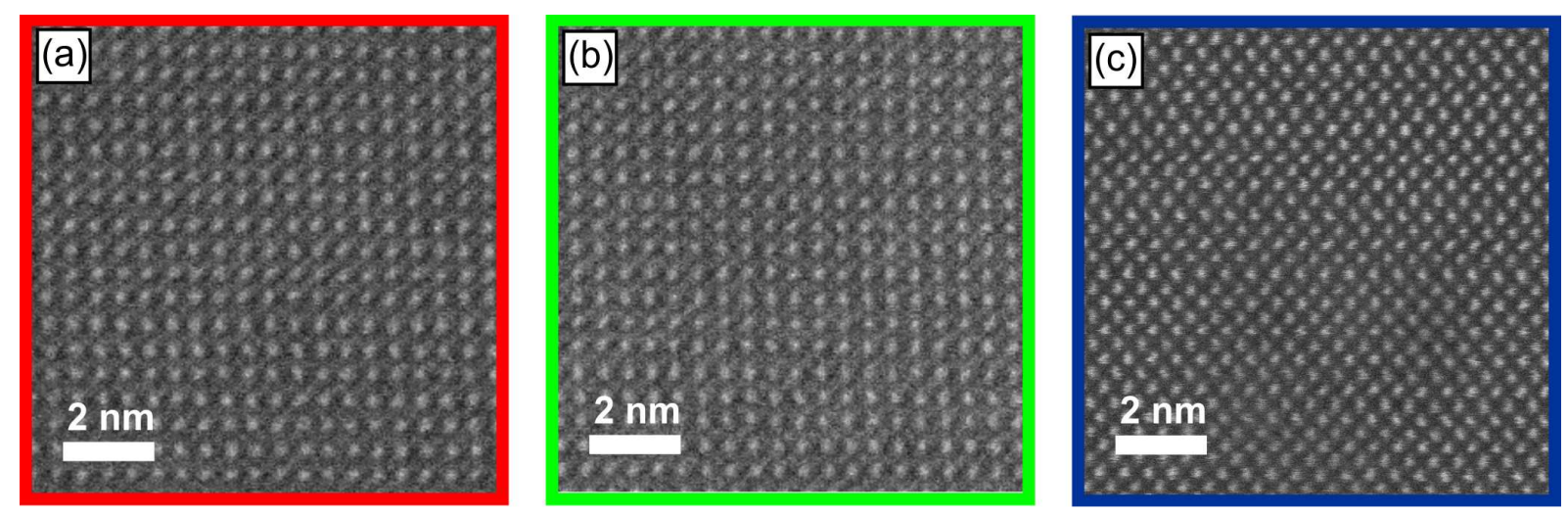

Figure 1. HAADF images of GaP (a), GaPAs (b) and GaAs (c) with comparable sample thickness of $30 \mathrm{~nm}$.
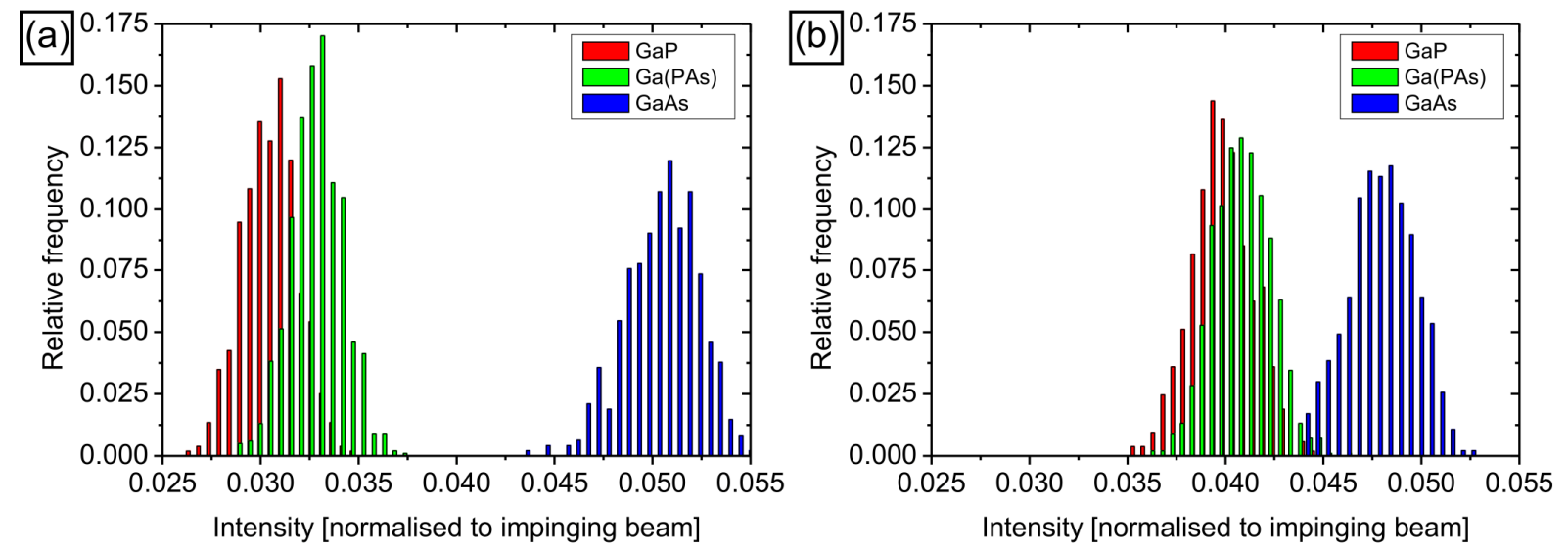

Figure 2. Statistical evaluation of the HAADF intensities on the group V (a) and group III lattices (b). 